\title{
Magnetic Field Configuration at the Galactic Center Investigated by Wide Field Near-Infrared Polarimetry: Transition from a Toroidal to a Poloidal Magnetic Field
}

\author{
Shogo Nishiyama ${ }^{1}$, Hirofumi Hatano ${ }^{2}$, Motohide Tamura ${ }^{3}$, Noriyuki Matsunaga ${ }^{4}$, Tatsuhito \\ Yoshikawa $^{1}$, Takuya Suenaga ${ }^{5}$, James H. Hough ${ }^{6}$, Koji Sugitani ${ }^{7}$, Takahiro Nagayama ${ }^{2}$, \\ Daisuke Kato $^{8}$, and Tetsuya Nagata ${ }^{1}$
}

\begin{abstract}
We present a large-scale view of the magnetic field in the central $2^{\circ} \times 2^{\circ}$ region of our Galaxy. The polarization of point sources has been measured in the $J$, $H$, and $K_{S}$ bands using the near-infrared polarimetric camera SIRPOL on the $1.4 \mathrm{~m}$ telescope IRSF. Comparing the Stokes parameters between high extinction stars and relatively low extinction ones, we obtain polarization originating from magnetically aligned dust grains in the central few-hundred pc of our Galaxy. We find that near the Galactic plane, the magnetic field is almost parallel to the Galactic plane (i.e., toroidal configuration) but at high Galactic latitudes $(|b|>$ 0.4 ), the field is nearly perpendicular to the plane (i.e., poloidal configuration). This is the first detection of a smooth transition of the large-scale magnetic field configuration in this region.
\end{abstract}

\footnotetext{
${ }^{1}$ Department of Astronomy, Kyoto University, Kitashirakawa-oiwake-cho, Sakyo-ku, Kyoto 606-8502, Japan; shogo@kusastro.kyoto-u.ac.jp

${ }^{2}$ Department of Astrophysics, Nagoya University, Furo-cho, Chikusa-ku, Nagoya 464-8602, Japan

${ }^{3}$ National Astronomical Observatory of Japan, 2-21-1, Osawa, Mitaka, Tokyo 181-8588, Japan

${ }^{4}$ Kiso Observatory, Institute of Astronomy, University of Tokyo, 10762-30, Mitake, Kiso, Nagano 3970101, Japan

${ }^{5}$ Department of Astronomical Sciences, Graduate University for Advanced Studies (Sokendai), 2-21-1, Osawa, Mitaka, Tokyo 181-8588, Japan

${ }^{6}$ Centre for Astrophysics Research, Science and Technology Research Institute, University of Hertfordshire, Hatfield AL10 9AB, UK

${ }^{7}$ Graduate School of Natural Sciences, Nagoya City University, 1, Yamanohata, Mizuho-cho, Mizuho-ku, Nagoya 467-8501, Japan

${ }^{8}$ Institute of Space and Astronomical Science, Japan Aerospace Exploration Agency, 3-1-1, Yoshinodai, Chuo-ku, Sagamihara, Kanagawa, 252-5210, Japan
} 


\section{INTRODUCTION}

It has long been a subject of controversy whether the magnetic field (MF) in the central region of our Galaxy has a dipolar or toroidal geometry on a large scale. Previous observations have been mainly based on sub-millimeter polarimetric observations of dense molecular clouds, or radio observations of non-thermal radio filaments. The former show that the MFs in the dense molecular clouds run almost parallel to the Galactic plane, indicating a toroidal MF (Novak et al. 2003; Chuss et al. 2003). By contrast, the radio filaments are aligned nearly perpendicular to the Galactic plane (Yusef-Zadeh et al. 1984, 2004; LaRosa et al. 2004), and are highly polarized along the filaments' long axes (Tsuboi et al. 1986; Yusef-Zadeh \& Morris 1987), suggesting a large-scale, pervasive poloidal MF in the intercloud medium.

The MFs both in dense molecular clouds and in the diffuse interstellar (intercloud) medium are observable via optical and near-infrared (NIR) polarimetry of intrinsically unpolarized stars with the polarization attributed to differential extinction by dust grains aligned with their short axis along the direction of the MF (e.g., Mathewson \& Ford 1970). The polarization vectors of such stars trace the orientation of the MF projected onto the plane of the sky, and measurements of stars at different distances reveal the change of the projected MF orientation along the line of sight. Although there are numerous stars near the Galactic center (GC), high extinction by the intervening dust grains means that infrared polarimetry is ideal (e.g., Tamura et al. 1987) in understanding the MF configuration in the GC. However, infrared polarimety toward the GC has been carried out for only a limited

number of stars so far (e.g., Hough et al. 1978; Kobayashi et al. 1983; Bailey et al. 1984; Eckart et al. 1995; Ott et al. 1999).

In this letter, we report observations of the GC region with a wide-field NIR polarimeter. In the first paper of this series (Nishiyama et al. 2009), we presented the MF configuration in the $20^{\prime} \times 20^{\prime}$ region of the GC. In the following observations from 2007, more than 300,000 stars are detected in the central $2^{\circ} \times 2^{\circ}$ region with an error in the degree of polarization less than $1 \%$. The statistical treatment of the large number of stars enables us to obtain the first large-scale view of the MF in the GC.

\section{OBSERVATIONS AND DATA REDUCTION}

Polarimetry for a large number of stars in the central $2^{\circ} \times 2^{\circ}$ region of the Galaxy was obtained with IRSF/SIRPOL between July 2006 and August 2009. SIRPOL consists of a single-beam polarimeter (a half-wave plate rotator unit and a fixed wire-grid polarizer; Kandori et al. 2006) and the NIR imaging camera SIRIUS (Simultaneous Infrared Imager for 
Unbiased Survey; Nagashima et al. 1999; Nagavama et al. 2003), and is attached to the 1.4$\mathrm{m}$ telescope IRSF (Infrared Survey Facility). The detectors are three $1024 \times 1024 \mathrm{HgCdTe}$ arrays, with a scale of $0^{\prime \prime} .45$ pixel $^{-1}$. SIRPOL provides images of a $7.7 \times 7$. 7 area of sky in three NIR bands, $J(1.25 \mu \mathrm{m}), H(1.63 \mu \mathrm{m})$, and $K_{S}(2.14 \mu \mathrm{m})$, simultaneously. The filter system of IRSF/SIRPOL is similar to the Mauna Kea Observatory system (Tokunaga 2000).

10-s exposures were made, at each 4 wave plate angles $\left(0 \circ 0,22^{\circ} .5,45^{\circ} .0,67^{\circ} .5\right)$, and at each of 10 dithered positions. The weather condition was photometric, with a mean seeing of $\sim 1^{\prime \prime} .3(J), \sim 1^{\prime \prime} .2(H)$, and $\sim 1^{\prime \prime}$. $1\left(K_{S}\right)$. Twilight flats were obtained before and after the observations. The IRAF (Image Reduction and Analysis Facility) 1 software package was used to perform dark- and flat-field corrections, followed by sky background estimation and subtraction.

\section{ANALYSIS}

To obtain the photometric magnitudes and errors, we used the DAOPHOT package (Stetson 1987). The sources detected in Stokes $I$ images $\left[I=\left(I_{0}^{\circ}+I_{22.5}+I_{45^{\circ}}+I_{67.5}\right) / 2\right]$ were input to the ALLSTAR task for PSF-fitting photometry. About 10 sources were used to construct the PSF in each image. Photometric calibration was performed with reference to the magnitudes of point sources in the 2MASS catalog (Skrutskie et al. 2006).

The Stokes parameters $I, Q$, and $U$ for point sources were determined from aperture polarimetry, which gives a better photometric result than PSF-fitting photometry in our GC data. We obtained the intensity for each wave plate angle $\left(I_{0}^{\circ}, I_{22.5}, I_{45^{\circ}}, I_{67^{\circ} .5}\right)$ with IRAF DAOFIND and APPHOT tasks. The size of the aperture is slightly different among fields due to variations of the seeing, and an aperture size of $1.5 \times$ FWHM was adopted, which was measured on Stokes $I$ images. Our data analysis results in $\sim 140,000(J), \sim 360,000(H)$, and $\sim 380,000\left(K_{S}\right)$ sources in the observed area with an error $\delta P$ in the degree of polarization $P$ less than $1 \%$, where

$$
P=\sqrt{\left(Q^{2}+U^{2}\right)} / I
$$

and

$$
\delta P=\frac{1}{P} \sqrt{\left(\frac{Q}{I}\right)^{2}\left[\delta\left(\frac{Q}{I}\right)\right]^{2}+\left(\frac{U}{I}\right)^{2}\left[\delta\left(\frac{U}{I}\right)\right]^{2}}
$$

\footnotetext{
${ }^{1}$ IRAF is distributed by the National Optical Astronomy Observatory, which is operated by the Association of Universities for Research in Astronomy, Inc., under cooperative agreement with the National Science Foundation.
} 
Polarimetric accuracy was checked with duplicate sources in overlapping regions of adjacent fields observed under different atmospheric condition. The means of the average differences of $Q / I$ and $U / I$ were $0.26 \%$ and $0.27 \%$ in the $H$ band, and $0.25 \%$ and $0.26 \%$ in the $K_{S}$ band, respectively.

To derive the MF configuration at the GC, we separated stars into two subgroups according to their colors: "blue" and "red" stars. Most of the stars detected in the observations are late-type giants near the $\mathrm{GC}$, and their intrinsic $H-K_{S}$ colors are almost the same2. So their observed colors depend on the amount of the interstellar extinction which in turn depends generally on the distance to the stars. The "red" ("blue") stars suffer strong (weak) extinction, and thus they are distributed at the far (near) side of the GC. The light from stars at the near side of the GC (i.e., "blue" stars) is transmitted through the interstellar dust in the Galactic disk, and the polarization is produced there, whereas the light from stars at the far side of the GC (i.e., "red" stars) is transmitted through the dust in the disk and the GC. By comparing the Stokes parameters $(Q / I$ and $U / I)$ of the "blue" and "red" stars, we obtain polarization of the GC component (see Nishivama et al. 2009, for more detail).

As a first step in deriving the MF configuration at the GC, the field was divided into sub-fields of $2^{\prime} \times 2^{\prime}$, and $H-K_{S}$ histograms were made for each sub-field. These histograms were fitted with the Gaussian function, and means $\left(m_{H-K_{S}}\right)$ and $\operatorname{sigmas}\left(\sigma_{H-K_{S}}\right)$ were calculated for each sub-field. Using the mean and sigma values, the stars were divided into two subgroups: "blue" stars with the color of $\left(m_{H-K_{S}}-2 \sigma_{H-K_{S}}\right)<\left(H-K_{S}\right)<m_{H-K_{S}}$, and "red" stars with $m_{H-K_{S}}<\left(H-K_{S}\right)<\left(m_{H-K_{S}}+2 \sigma_{H-K_{S}}\right)$.

Mean $\left(m_{H-K_{S}}\right)$ and sigma $\left(\sigma_{H-K_{S}}\right)$ in the $H-K_{S}$ histograms were determined for stars with $H<15.5$, which are bright enough to avoid the influence of the limiting magnitude, $H \approx 16.6$ (see also Fig. 7 of Nishiyama et al. 2009). This means that our observations are equally sensitive to stars on the far and near sides of the GC, and using this criterion, stars on both sides of the GC can be distinguished. The "blue" and "red" stars are in fact distributed evenly in the $2^{\prime} \times 2^{\prime}$ sub-fields. Hence, it can be safely assumed that the peaks in the $H-K_{S}$ histograms correspond to a real peak of spatial distribution of stars along the line of sight.

Next, $Q / I$ and $U / I$ histograms in the $K_{S}$ band were constructed for the "blue" and "red" stars with $\delta P<3 \%$ in each sub-field. Their means, $<Q / I>_{\mathrm{B}},<Q / I>_{\mathrm{R}},<U / I>_{\mathrm{B}}$,

\footnotetext{
${ }^{2}$ The standard deviation of the intrinsic $H-K_{S}$ colors of the observed giants in the GC is calculated to be 0.06 from the Galactic model by Wainscoat et al. (1992). This is small enough compared to the mean of the color ranges of red and blue stars, $2 \sigma_{H-K_{S}}=0.38$. Therefore we proceed on the supposition that the observed colors of the giants depend on the distance to them.
} 
and $<U / I>_{\mathrm{R}}$, were used to obtain the degree $P$ and position angle $\theta$ of polarization for "red" minus "blue" (i.e., GC component) with the following equations (Goodrich 1986):

$$
\begin{gathered}
P_{\mathrm{R}-\mathrm{B}}=\sqrt{\left(\left\langle\frac{Q}{I}\right\rangle_{\mathrm{R}}-\left\langle\frac{Q}{I}\right\rangle_{\mathrm{B}}\right)^{2}+\left(\left\langle\frac{U}{I}\right\rangle_{\mathrm{R}}-\left\langle\frac{U}{I}\right\rangle_{\mathrm{B}}\right)^{2}}, \\
\theta_{\mathrm{R}-\mathrm{B}}=\frac{1}{2} \arctan \left(\frac{\left\langle\frac{U}{I}\right\rangle_{\mathrm{R}}-\left\langle\frac{U}{I}\right\rangle_{\mathrm{B}}}{\left\langle\frac{Q}{I}\right\rangle_{\mathrm{R}}-\left\langle\frac{Q}{I}\right\rangle_{\mathrm{B}}}\right) .
\end{gathered}
$$

The errors of $\langle Q / I\rangle$ and $\langle U / I\rangle$ were calculated from the standard error on the mean $\sigma / \sqrt{N}$ of the $Q / I$ and $U / I$ histograms, where $\sigma$ is the standard deviation and $N$ is the number of stars. A few sub-fields with strong extinction are excluded from our analysis. We confirmed that the ratio of the number of the "red" and "blue" stars in each sub-field has a mean of $\approx 1$ even near the Galactic plane $(l<0.2)$, suggesting no bias effect to detect more stars at the near side than the far side of the GC due to strong extinction. Finally, $\langle Q / I\rangle$ and $\langle U / I\rangle$ are averaged in a circle of 2.4 radius with a 3.0 grid, and $P_{\mathrm{R}-\mathrm{B}}$ and $\theta_{\mathrm{R}-\mathrm{B}}$ are calculated with the equations (11) and (2). The resultant mean and rms of $P_{\mathrm{R}-\mathrm{B}}$ are $0.29 \%$ and $0.12 \%$ in the $K_{S}$ band, respectively.

The strong concentration of stars towards the GC allows measurement of the MF structure in the GC region. Most of the stars detected in our observations have an intrinsic color of red giants with spectral type of $\mathrm{K}$ and M. We used a Galactic model (Wainscoat et al. 1992) to show the degree of central concentration of the stars along the line of sight, and found that more than $85 \%$ of stars within the central $2^{\circ} \times 2^{\circ}$ region are expected to be within $300 \mathrm{pc}$ of the GC. So the observed position angles of polarization of the GC component show those of MFs, averaged along the lines of sight, in the central few-hundred pc.

\section{RESULTS AND DISCUSSION}

Fig. 1 shows the MF direction ( $E$-vectors of polarization of the GC component in the $K_{S}$ band) in the GC. This is the first large-scale view of the MF in this region. The MF directions in the dense molecular clouds, measured with sub-millimeter (sub-mm) and farinfrared (FIR) polarimetry, are overplotted on the $K_{S}$ data in Fig. 2. The panel (a) shows the MF directions derived from polarimetry in a large survey at $450 \mu \mathrm{m}$ (Novak et al. 2003). Near the Sagittarius A region, sub-mm polarimetry has been carried out with better spatial resolution (Fig. 2 b; Dotson et al. 2000; Novak et al. 2000; Chuss et al. 2003). In both cases, the MF directions derived from the sub-mm/FIR polarimetry are well matched with the NIR 
observations. It follows that the position angle of polarization derived from NIR polarimetry can be used to determine the direction of the MF in the GC.

The MF direction has strong dependence on the Galactic latitude, i.e., distance from the Galactic plane, but not on the Galactic longitude (Fig. 3). The MF direction (i.e., the position angle of polarization) is defined as the angular offset in degrees from north through east in Galactic coordinates. The histogram of the MF direction at $|b| \leq 00^{\circ} 4$ has a clear peak of $\sim 90^{\circ}$, suggesting a toroidal MF configuration in this region. At higher Galactic latitude $\left(|b|>00^{\circ} 4\right)$, the mean MF direction appears to swing from $90^{\circ}$ to $\sim 170^{\circ}$ which is nearly perpendicular to the Galactic plane. These results suggest a transition of the large-scale MF configuration from toroidal to poloidal in the GC region, at $|b| \sim 0.4$.

In the central few hundred pc region, there are some structures surrounding the GC, such as the 180-pc (or expanding) molecular ring (Kaifu et al. 1972; Scoville 1972), and the GC molecular arms composing a ring of radius of $\sim 120$ pc (Sofue 1995). It is therefore difficult to completely exclude the possibility that we are preferentially probing magnetic fields in these molecular structures. However, it seems to be convincing that we have detected the magnetic fields in molecular clouds much closer to the $\mathrm{GC}$, from the following points. As shown in Fig. 2 (see also Fig. 10 of Nishiyama et al. 2009), we are able to replicate the detailed structure of the sub-mm/FIR magnetic field maps in the circumnuclear disk, and two molecular clouds $\mathrm{M}-0.02-0.07\left(+50 \mathrm{~km} \mathrm{~s}^{-1}\right.$ cloud $)$ and $\mathrm{M}-0.13-0.08\left(+20 \mathrm{~km} \mathrm{~s}^{-1}\right.$ cloud). These two molecular clouds are located within several tens of pc from the GC, and the possibility of contribution of foreground emission was also ruled out (Novak et al. 2000). Hence we can probe the magnetic fields very close to the GC.

Previously, the MF configuration of the GC has been viewed as poloidal in the diffuse, interstellar (intercloud) medium, and approximately parallel to the Galactic plane only in the dense molecular clouds (e.g., Ferrière 2009). The new data presented here shows a toroidal MF prevails at $|b|<0$, , even outside the dense molecular clouds (Fig. 2), which radio (Oka et al. 1998) and sub-mm (Pierce-Price et al. 2000) surveys show are mostly confined to within $\sim 0.2$ of the Galactic plane. Toward higher Galactic latitudes $(|b|>0.4)$, the field changes from a toroidal to a poloidal configuration.

Since SIRPOL also provides images in the $H$ band, it is possible to compare these with the $K_{S}$-band results (Fig. 1). Most of the position angles of polarization in the $H$ band show the same direction as that in the $K_{S}$ bands within their errors. In addition, there is a good correlation between the degree of polarization $P$ in the $H$ and $K_{S}$ bands with $P_{K_{S}}=0.611 \times P_{H}$. This corresponds to $\beta=1.85$ for a power-law approximation $P_{\lambda} \propto \lambda^{-\beta}$ which represents the wavelength dependence of interstellar polarization at NIR wavelengths, consistent with previous results toward the $\mathrm{GC}(\beta \approx 1.8-2.0$; Nagata et al. 
1994; Hatano et al. 2010). The correlation coefficients in the degree and position angle of polarization are 0.71 and 0.92 , respectively, showing strong correlation between the $H$ and $K_{S}$ bands. These results strongly suggest that the differential extinction by magnetically aligned dust grains is responsible for the observed NIR polarization.

The existence of the toroidal MF even in the intercloud medium near the Galactic plane is consistent with the observations of Galactic center diffuse X-ray (GCDX) emission (Koyama et al. 1989). The spatial distribution of the $6.7 \mathrm{keV}$ line emission from Helium-like ions of iron is elliptical with a major axis approximately along the Galactic plane (Yamauchi et al. 1990). Although the origin of the GCDX is as yet unclear, recent observations with the Suzaku satellite found a smooth and monotonic variation of the ionization temperature along the Galactic longitude, and a clear difference of distribution between the $6.7 \mathrm{keV}$ line flux and an integrated point source flux, suggesting hot, diffuse interstellar plasma in origin (Kovama et al. 2007). If a poloidal MF is pervasive in this region, since the gravitational well of the GC region is far too shallow to confine them, the hot plasma must be rushing out of the plane vertically as a galactic wind. Such a vertical extension of the GCDX has not been observed.

The large-scale MF configuration has important implications for understanding the origin of a variety of magnetic phenomena. The double helix nebula (Morris et al. 2006) extends almost perpendicular to the Galactic plane from $b=0.5$ to 0.8 . It exists in the region of the large-scale poloidal MF, so the nebula probably results from a dynamically ordered interstellar phenomenon involving its local interstellar MFs. On the other hand, a large part of the non-thermal radio filaments exist at $|b| \lesssim 0^{\circ} 4$ where the toroidal field is dominant (Yusef-Zadeh et al. 2004; LaRosa et al. 2004). Hence the filaments do not run along the MF directions we obtained; rather, they are almost perpendicular to the local MF. This implies that it is important to reconsider the simple picture in which the non-thermal filaments are illuminated flux tubes within a uniform poloidal MF (Morris 1990), for the origin of the filaments.

We are grateful to T. Oka for kindly providing the ${ }^{12} \mathrm{CO} J=1-0$ data. We thank the staff of the South African Astronomical Observatory (SAAO) for their support during our observations. S. N. and H. H. are financially supported by the Japan Society for the Promotion of Science (JSPS) through the JSPS Research Fellowship for Young Scientists. This work was partly supported by the Grants-in-Aid for Young Scientists (B) 19740111, Scientific Research (C) 21540240, and the Global COE Program "The Next Generation of Physics, Spun from Universality and Emergence" from the Ministry of Education, Culture, Sports, Science and Technology (MEXT) of Japan. M. T. has been supported by the MEXT, Grants-in-Aid 19204018 and 22000005. This publication makes use of data from the Two Micron All Sky 
Survey, a joint project of the University of Massachusetts, the Infrared Processing and Analysis Center, the National Aeronautics and Space Administration, and the National Science Foundation.

\section{REFERENCES}

Bailey, J., Hough, J. H., \& Axon, D. J. 1984, MNRAS, 208, 661

Chuss, D. T., et al. 2003, ApJ, 599, 1116

Dotson, J. D., Davidson, J., Dowell, C. D., Schleuning, D. A., \& Hildebrand, R. H. 2000, ApJS, 128, 335

Eckart, A., Genzel, R., Hofmann, R., Sams, B. J., \& Tacconi-Garman, L. E. 1995, ApJ, 445, L23

Ferrière, K. 2009, A\&A, 505, 1183

Goodrich, R. W. 1986, ApJ, 311, 882

Hatano, H., et al. 2010, AJ, submitted

Hough, J. H., McCall, A., Adams, D. J., \& Jameson, R. F. 1978, A\&A, 69, 431

Kandori, R. et al. 2006, Proc. SPIE, 6269, 159

Kaifu, N., Kato, T., \& Iguchi, T. 1972, Nature, 238, 105

Kobayashi, Y., Okuda, H., Sato, S., Jugaku, J., \& Dyck, H. M. 1983, PASJ, 35, 101

Koyama, K., Awaki, H., Kunieda, H., Takano, S., \& Tawara, Y. 1989, Nature, 339, 603

Koyama, K., et al. 2007, PASJ, 59, 245

LaRosa, T. N., Nord, M. E., Joseph, T., Lazio, W., \& Kassim, N. E. 2004, ApJ, 607, 302

Mathewson, D. S., \& Ford, V. L. 1970, Mem. R. astr. Soc. 74, 139

Morris, M., 1990 in Galactic and intergalactic magnetic fields, (eds R. Beck, R. Wielebinski, \& P. P. Kronberg) 361 (Proc. IAU Symp. 140)

Morris, M., Uchida, K., \& Do, T. 2006, Nature, 440, 308 
Nagashima, C. et al. in Star Formation 1999, ed. T. Nakamoto 1999, (Nobeyama : Nobeyama Radio Obs.), 397

Nagata, T., Kobayashi, N., \& Sato, S. 1994, ApJ, 423, L113

Nagayama, T. et al. 2003, Proc. SPIE, 4841, 459

Nishiyama, S., et al. 2009, ApJ, 690, 1648

Novak, G., Dotson, J. L., Dowell, C. D., Hildebrand, R. H., Renbarger, T., \& Schleuning, D. A. 2000, ApJ, 529, 241

Novak, G., Chuss, D. T., Renbarger, T., Griffin, G. S., Newcomb, M. G., Peterson, J. B., Loewenstein, R. F., Pernic, D., \& Dotson, J. L. 2003, ApJ, 583, L83

Oka, T., Hasegawa, T., Sato, F., Tsuboi, M., \& Miyazaki, A. 1998, ApJS, 118, 455

Ott, T., Eckart, A., \& Genzel, R. 1999, ApJ, 523, 248

Pierce-Price, D. et al. 2000, ApJ, 545, L121

Scoville, N. Z. 1972, ApJ, 175, L127

Skrutskie, M. F., et al. 2006, AJ, 131, 1163

Stetson, P. B. 1987, PASP, 99, 191

Sofue, Y. 1995, PASJ, 47, 527

Tamura, M., Nagata, T., Sato, S., \& Tanaka, M. 1987, MNRAS, 224, 413

Tokunaga, A. T. 2000, Astrophysical Quantities, 4th edition, ed. Cox, A. (AIP Press), p.143

Tsuboi, M., Inoue, M., Handa, T., Tabara, H., Kato, T., Sofue, Y., \& Kaifu, N. 1986, AJ, 92, 818

Wainscoat, R. J., Cohen, M., Volk, K., Walker, H. J., \& Schwartz, D. 1992, ApJS, 83, 111

Yamauchi, S., Kawada, M., Koyama, K., Kunieda, H., \& Tawara, Y. 1990, ApJ, 365, 532

Yusef-Zadeh, F., Morris, M., \& Chance, D. 1984, Nature, 310, 557

Yusef-Zadeh, F., \& Morris, M. 1987, ApJ322, 721

Yusef-Zadeh, F., Hewitt, J. W., \& Cotton, W. 2004, ApJS, 155, 421 


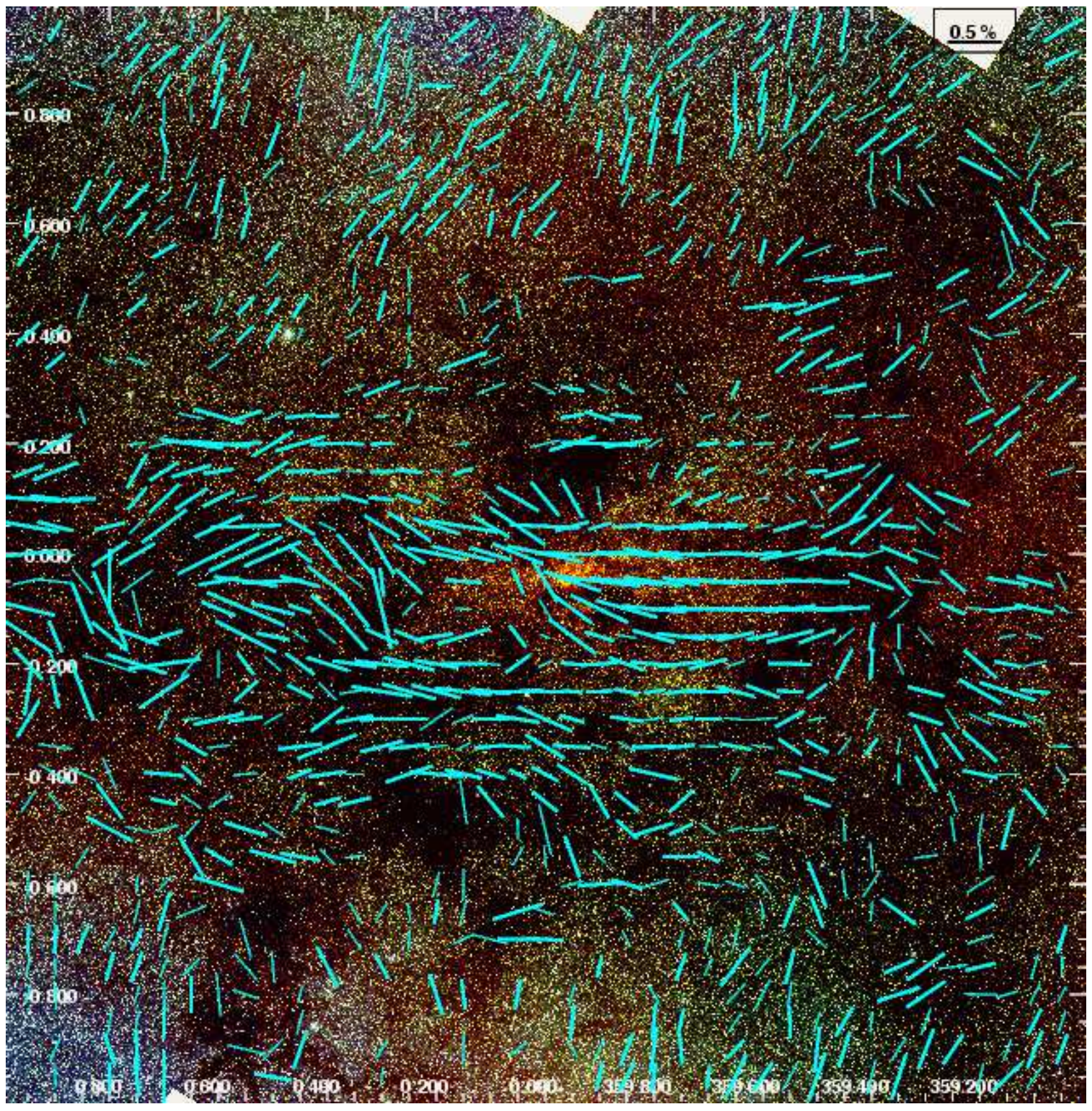

Fig. 1. - NIR mosaic image of the Galactic center region covering $2.0 \times 2.0$ in the Galactic coordinate, taken with the IRSF telescope and NIR camera SIRIUS. The three NIR bands are $J$ (blue, $1.25 \mu \mathrm{m}), H$ (green, $1.63 \mu \mathrm{m})$, and $K_{S}$ (red, $\left.2.14 \mu \mathrm{m}\right)$. The central-parsec star cluster of the Galactic center is the bright yellow blob in the center of the image. Observed $E$-vectors of polarization for the Galactic center components in the $K_{S}$ band are also plotted with cyan bars whose length indicates the degree of polarization. The vectors are averaged in a circle of 2.4 radius with a $3^{\prime} .0$ grid, and plotted with thick bars (detected with more than $3 \sigma$ ) and thin bars (detected with $2-3 \sigma$ ). 


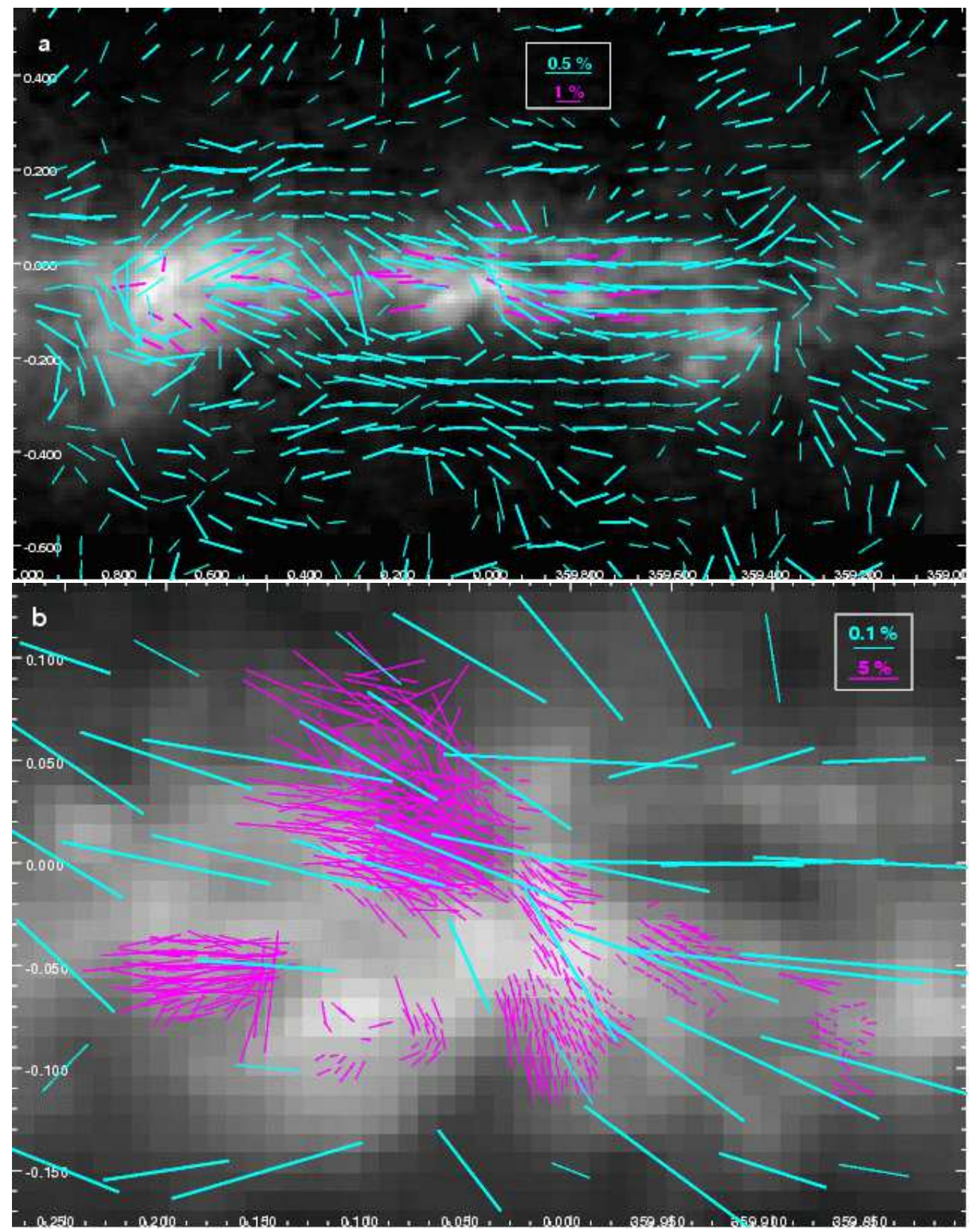

Fig. 2.- Maps of the ${ }^{12} \mathrm{CO} J=1-0$ line emission integrated over the velocity range $V_{\mathrm{LSR}}=-100$ to $+100 \mathrm{~km} \mathrm{~s}^{-1}$ in Galactic coordinate (Oka et al. 1998). Pink bars in the panel (a) are drawn parallel to the magnetic field direction derived from polarimetry at $450 \mu \mathrm{m}$ (Novak et al. 2003), and those in the panel (b) from measurements at $60 \mu \mathrm{m}, 100$ $\mu \mathrm{m}$ (Dotson et al. 2000), and $350 \mu \mathrm{m}$ (Novak et al. 2000). The MF directions measured at near-infrared wavelength (same as Fig. 1) are also plotted with cyan bars whose length indicates the degree of polarization. Note that since emissive polarization in the sub-mm/FIR wavelengths is perpendicular to the magnetic field, vectors orthogonal to it are shown here. 

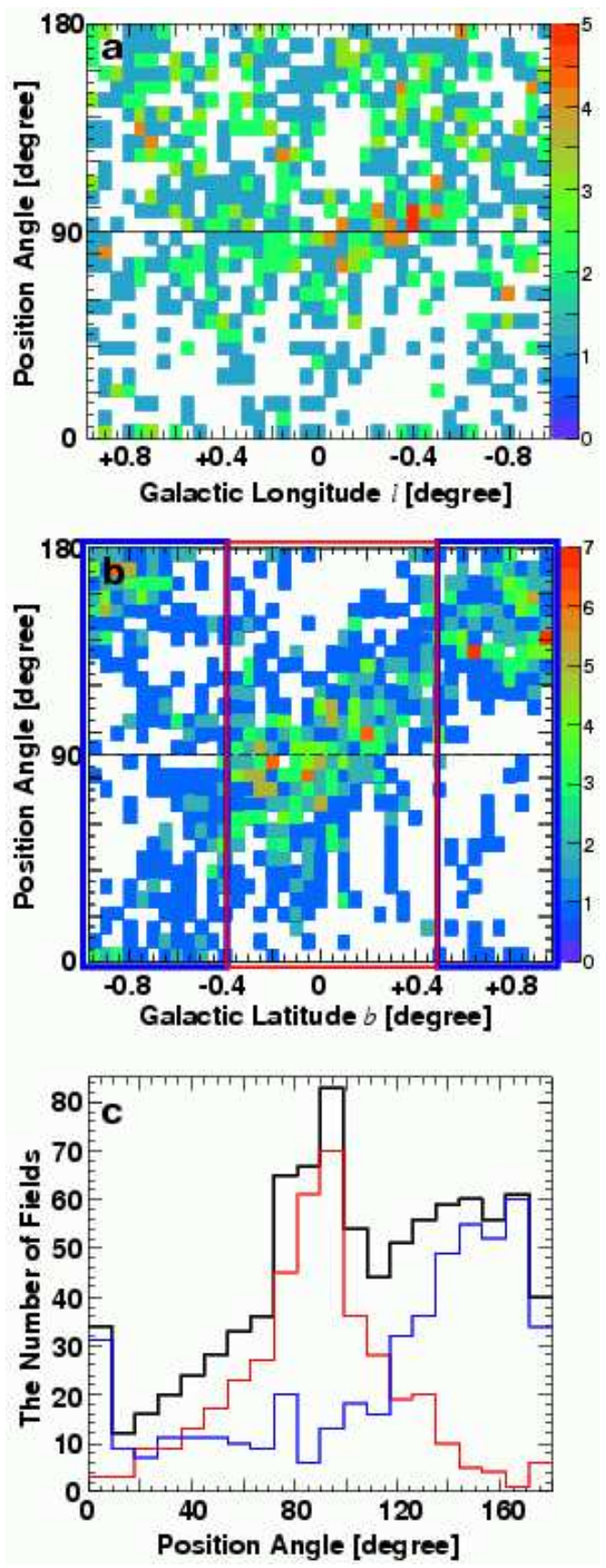

Fig. 3.- Directions of the magnetic fields in the Galactic center as a function of the Galactic longitude (a) and latitude (b). The number of field per bin is shown in different colors, as indicated in the color scale. The MF direction is defined as the angular offset in degrees from north through east in Galactic coordinates. The magnetic field swings from the direction along the Galactic plane at $b \sim 0^{\circ}$ to the direction nearly perpendicular to the plane at $|b| \sim 1$ 1.0. Histograms of the magnetic field direction at $|b| \leq 0^{\circ} .4$ (red), at $|b|>0.4$ (blue), and both (black) are also shown in the panel (c). The red histogram has a clear peak at the direction almost parallel to the Galactic plane, while the blue one has a peak at $\sim 170^{\circ}$, almost perpendicular to the plane. 